OPEN ACCESS

Edited by:

Andrea Ladányi,

National Institute of Oncology (NIO),

Hungary

${ }^{*}$ Correspondence:

Xu Hou

houxu1983@hotmail.com

Ming Gao

gaomingsjwk@163.com

${ }^{\dagger}$ These authors have contributed equally to this work

Received: 29 March 2021 Accepted: 27 August 2021 Published: 22 September 2021

Citation:

Wang X, Yang Q, Liu N, Bian Q, Gao M and Hou X (2021) Clinical Value of TXNDC12 Combined With IDH and $1 p 19 q$ as Biomarkers for Prognosis

of Glioma.

Pathol. Oncol. Res. 27:1609825. doi: 10.3389/pore.2021.1609825

\section{Clinical Value of TXNDC12 Combined With IDH and 1p19q as Biomarkers for Prognosis of Glioma}

\author{
Xinzhuang Wang ${ }^{1 \dagger}$, Quan $\mathrm{Yang}^{2 \dagger}$, Nan $\mathrm{Liu}^{2 \dagger}$, Qilong Bian ${ }^{3}$, Ming $\mathrm{GaO}^{2 *}$ and $\mathrm{Xu} \mathrm{Hou}{ }^{2 *}$ \\ ${ }^{1}$ Department of Neurosurgery, First Affiliated Hospital, Zhengzhou University, Zhengzhou, China, ${ }^{2}$ Department of Neurosurgery, \\ The First Affiliated Hospital of Harbin Medical University, Harbin, China, ${ }^{3}$ Department of Neurosurgery, Heze Municipal Hospital, \\ Heze, China
}

Background: Glioma is the primary malignant tumor of the central nervous system and presents high mortality and disability rates under existing treatment measures. Thioredoxin domain-containing 12 (TXNDC12) has been shown to play an important role in various malignant tumors. Therefore, we explored the clinicopathological characteristics of TXNDC12 in glioma to bring to light new ideas in its treatment.

Methods: We obtained data packages related to TXNDC12 expression status in gliomas from public databases. We analyzed glioma TXNDC12 expression and patient survival status and validated the above results using glioma specimens from our institution. Next, we analyzed the value of TXNDC12 in combination with $1 \mathrm{p} 19 \mathrm{q}$ and isocitrate dehydrogenase $(\mathrm{IDH})$ on the prognosis of glioma by regression model and receiver operating characteristic curve (ROC). Finally, we explored the function of related genes by $\mathrm{GO}$ analysis and KEGG analysis.

Results: Compared with normal brain tissue, the expression of TXNDC12 in glioma cells, regarding both mRNA and protein levels, was significantly upregulated. The survival time of patients with high-expression of TXNDC12 in glioma cells was shortened. In the World Health Organization pathological classification, IDH status, 1p19q status, and IDH combined with 1p19q subgroups, the expression of TXNDC12 increased with the deterioration of the above indicators. Tumor local immune analysis showed that the immune cell infiltration in TXNDC12 high-expressing glioma tissue increased, the tumor purity was reduced. GO and KEGG analyses indicated that TXNDC12 may be involved in the malignant prognosis of glioma through glycosylation and antigen processing and presentation.

Conclusion: We showed that TXNDC12 is significantly highly expressed in gliomas. This high expression predicts the poor prognosis of glioma patients and is related to the gliomas' local immune microenvironment. As a tumor-related gene, TXNDC12 may be used as a new prognostic judgment molecule.

Keywords: glioma, prognosis, TXNDC12, IDH, 1p19q 


\section{INTRODUCTION}

Gliomas account for $48.6 \%$ of all central nervous system (CNS) tumors and $81 \%$ of all primary CNS malignancies[1]. Even with the current standard of care, the median survival of newly diagnosed glioblastoma patients is only $12-15$ months[2,10], while the pathological progression of low-grade glioma is highly variable $[3,4]$. Eventually, most low-grade gliomas gradually evolve into high-grade gliomas[5]. It is therefore apparent that the course of different grades of gliomas varies greatly[6], and accurately distinguishing between them is particularly important. The discovery of molecular changes such as $1 \mathrm{p} 19 \mathrm{q}$ and IDH has brought major innovations to the diagnosis, treatment, and prognosis of glioma. Carrying IDH mutations or $1 \mathrm{p} 19 \mathrm{q}$ codeletion means that patients have a better prognosis[7,10]. However, IDH, and $1 \mathrm{p} 19 \mathrm{q}$ are mostly found in grade II and III gliomas, and rarely in grade IV gliomas[8,9], which are the most malignant. In addition, a variety of molecular and pathological pathways are involved in the formation and development of glioma $[10,11]$. The existing molecular targets have limited relevance to understanding the overall glioma pathogenesis and for guiding treatment. Therefore, it is necessary to develop new molecular markers to improve the classification criteria of glioma.

TXNDC12 is a member of the family of thioredoxin domains, currently known by other names, including ERp16, ERp18, ERp19, AGR1, and hTLP19[12-14]. TXNDC12 is ubiquitously expressed in all tissues and encodes a protein that is primarily involved in catalyzing the formation of disulfide bonds, showing similarities to protein-disulfide bond isomerase activity and playing an important role in endoplasmic reticulum stress[12]. In recent years, researchers have found that TXNDC12 mRNA also plays an important role in tumorigenesis and development[15-17]. For example, the expression level of TXNDC12 mRNA in gastric, liver, and other tumor tissues is significantly higher than that in nontumor tissues and is significantly associated with poor prognosis of patients[18,19]. TXNDC12 can promote hepatocellular carcinoma metastasis and invasion by upregulating the ZEB1-mediated epithelial-mesenchymal transition process and significantly shortening the postoperative survival of gastric cancer patients [20]. As another member of the thioredoxin family, TXNDC9 is upregulated in gliomas. Knockouts of the TXNDC9 gene can inhibit the proliferation and metastasis of glioma cells and induce apoptosis of glioma cells[21]. Therefore, we speculate that TXNDC12 may be involved in glioma development and may be used as a potential prognostic molecular marker.

Using multicenter and large-sample data, we demonstrated that TXNDC12 is highly expressed in glioma. Its overexpression closely correlates with glioma prognosis, tumor local immune status, IDH status, and 1p19q status, indicating that TXNDC12 may be a promising prognostic marker for glioma.

\section{MATERIAL AND METHODS}

\section{Obtainment of Clinical Samples}

This study was approved by the Ethics Committee of the First Affiliated Hospital of Harbin Medical University (No. HMUIRB-
2008-06), and the patients' consent was obtained. All glioma tissue specimens were taken from surgically resected tissues of patients with glioma $(n=25)$, and non-tumor brain tissue (From patients undergoing epilepsy surgery) was used as a negative control group $(n=5)$. Tissue samples were stored in liquid nitrogen.

\section{Download and Filtering of the Data Collection}

After screening out samples with defects in clinical information, we collected 546 glioma samples and five normal brain samples from The Cancer Genome Atlas (TCGA) (https://www.cancer.gov) and 749 glioma samples and 20 normal brain samples from the Chinese Glioma Genome Atlas (CGGA) (http://www.cgga.org.cn). The samples' clinical information is shown in Supplementary Table S1, which mainly included glioma grade, $1 \mathrm{p} 19 \mathrm{q}$ deletion status, IDH mutation status, age, and survival information.

\section{Bioinformatics Analysis of TXNDC12}

The differences in TXNDC12 expression between normal brain tissue and gliomas were examined by Wilcoxon test. The relationship between TXNDC12 expression and the clinical characteristics of glioma patients was analyzed using the Kruskal test or Wilcoxon test. Survival and ROC curves were plotted using the Survival and ROC Curves package. The scoring of sample immune gene sets was completed using GSVA, Limma, and GSEABase software packages, and an immune heatmap was created using heatmap software. The CIBERSORT R script v1.03 calculated the infiltration of immune cells in glioma samples. The median TXNDC12 expression in samples was used as a threshold to divide samples into high and low expression groups, and the Vioplot software package was used to depict the differences in immune cell infiltration between the two groups.

\section{Quantitative RT-PCR}

According to the manufacturer's instructions, the total RNA was extracted by TRIzol reagent (Carlsbad Invitrogen, California, United States). The total RNA concentration was detected using a NanoDrop2000 (Thermo Science ${ }^{\mathrm{TM}}$ ). RNA (1,000 ng) was reverse transcribed into cDNA using a qPCR RT Kit (TOYOBO, Japan). FastStart Universal 96 SYBR Green Master (ROX) (Roche, Germany) was used to detect the relative expression of TXNDC12 and housekeeping gene GAPDH by qRT-PCR. The primer sequences used in our procedure were as follows: TXNDC12, 5-GTCCTGCTGATTGTGAAAATGGC-3 and 5-TGATCCATGTCGAG GGTCAAA-3; GAPDH, 5-AAT CCCATCACCATCTTC-3 and 5-AGGC TGTTGTCATACTTC$3^{\prime}$. A non-paired t-test was performed between the two groups, and $p<0.05$ was considered statistically significant.

\section{Immunohistochemistry of TXNDC12}

We selected 3 normal brain tissues, 3 WHO III gliomas, and 3 WHO IV glioma specimens from the pre-embedded wax blocks, and sliced them into $5 \mu \mathrm{m}$ thick sections. The section tissue was deparaffinized with xylene, $3 \%$ hydrogen peroxide blocked the endogenous peroxidase activity, fetal bovine serum blocked at room temperature, and the primary antibody $\left(4^{\circ} \mathrm{C}\right.$, overnight $)$ and 
A

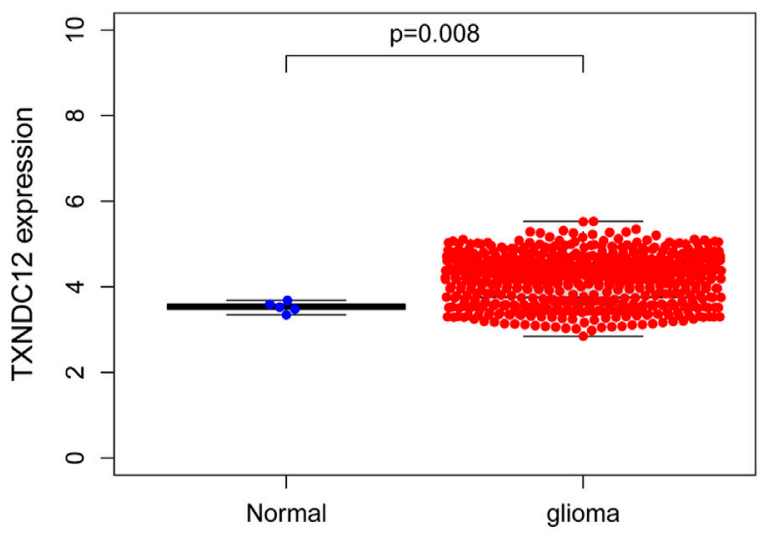

C

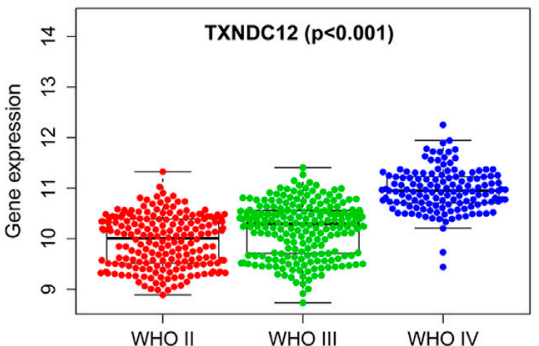

D

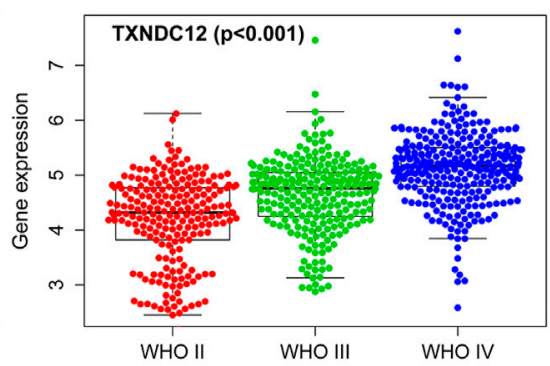

E

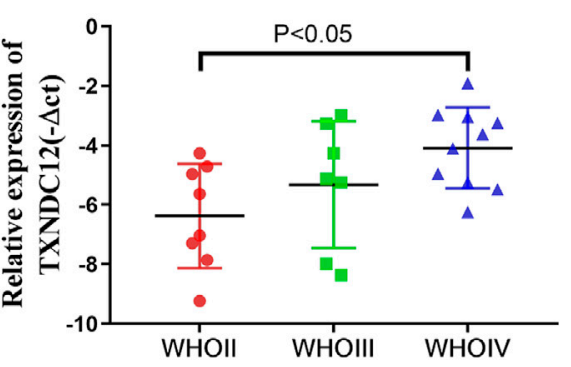

$\mathbf{F}$
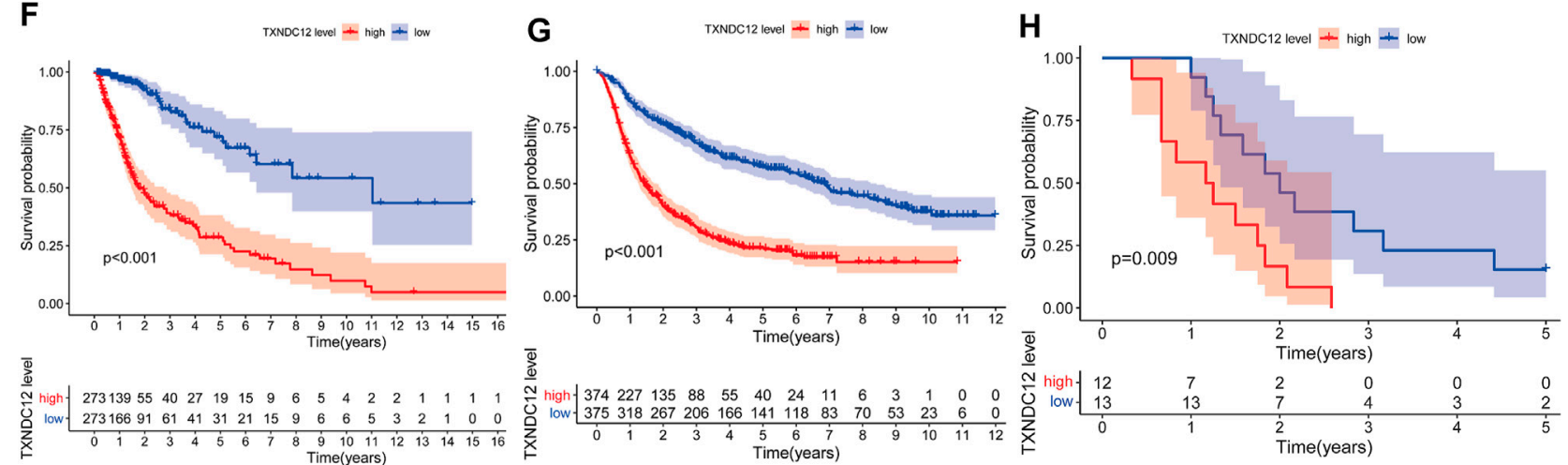

FIGURE 1 | TXNDC12 expression in TCGA (A) and surgical resection samples of glioma in our hospital (B). The relationship between TXNDC12 expression and WHO grade of glioma: TCGA (C), CGGA (D), and surgical resection samples of glioma in our hospital (E). Survival curves of glioma patients from TCGA (F), CGGA (G), and our hospital $\mathbf{( H )}$.

secondary antibody ( 2 h, room temperature) were incubated. Next, samples were visualized by using the diaminobenzidine (DAB) substrate kit. The primary antibody was TXNDC12 (A14403, ABclonal), and the secondary antibody was Goat Anti-Rabbit IgG (S0001, Affinity). Brown staining is a positive feature in cells.

\section{Enrichment Analysis of TXNDC12}

The differential genes that were co-expressed with TXNDC12 in samples (cor $>0.5$ ) were screened by cor.test function in the TCGA and CGGA databases, and the intersection was taken using a Venn diagram. The function of differential genes was analyzed using the $\mathrm{R}$ package (clusterProfiler, org.Hs.eg.db, enrichplot, ggplot2).

\section{Statistical Analysis}

$\mathrm{R} \times 64$ 3.5.2, strawberry-perl-5.30.2.1, and GraphPad Prism7 were used for plotting and statistics. $p<0.05$ was considered statistically significant.

\section{RESULTS \\ TXNDC12 is Associated With Poor Prognosis of Glioma Patients}

In samples from TCGA, TXNDC12 expression was significantly higher in glioma tissues compared to normal brain tissues 


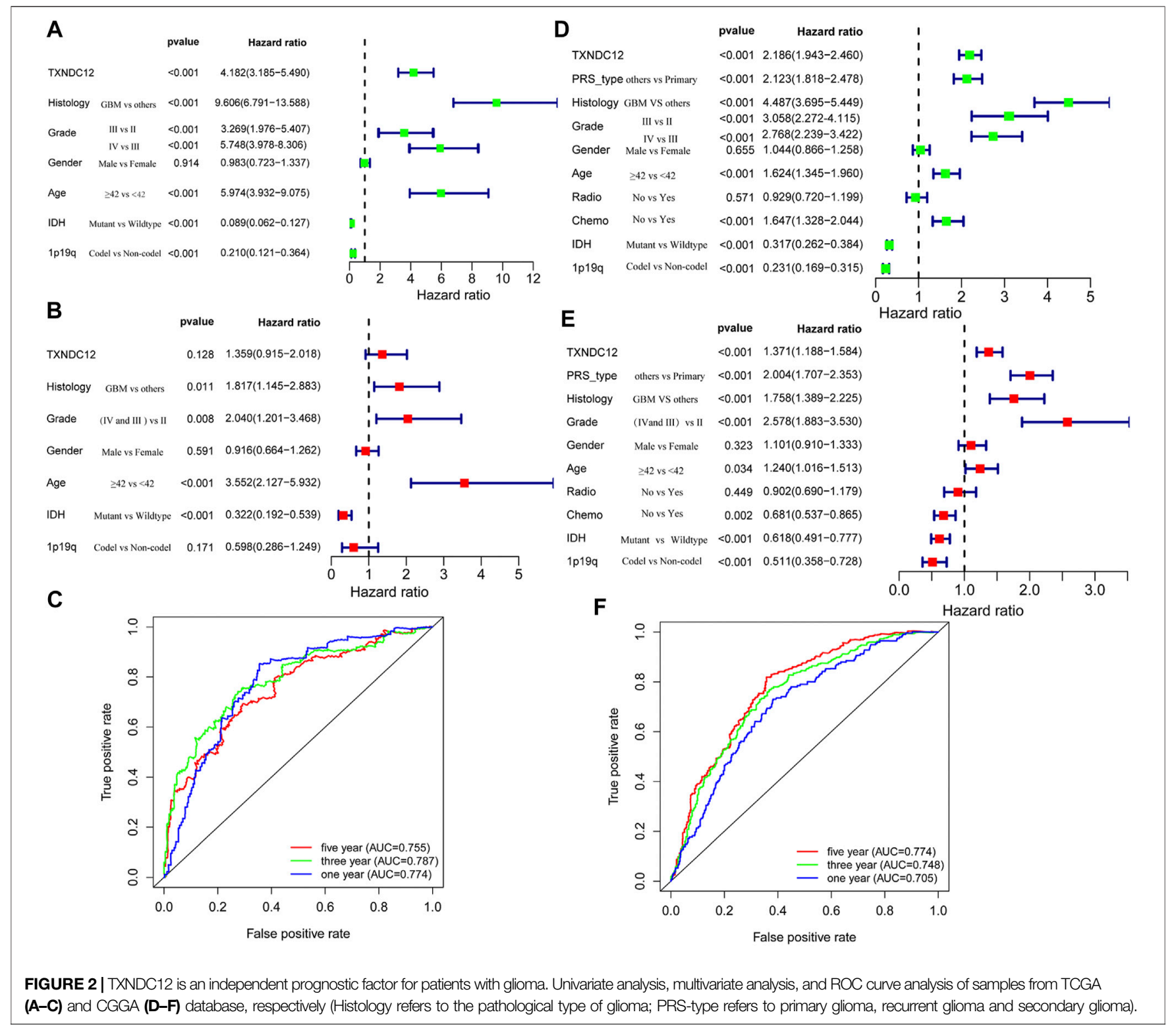

(Figure 1A, $p<0.01$ ). We further analyzed the relationship between TXNDC12 expression and glioma pathology grade. We found that TXNDC12 expression increased significantly as glioma grade increased, both in TCGA (Figure 1C, $p<0.01$ ) and CGGA (Figure 1D, $p<0.01$ ). Survival curve analysis showed that the prognosis of the TXNDC12 high expression group was poorer than that of the low expression group in both TCGA (Figure 1F, $p<0.01$ ) or CGGA (Figure 1G, $p<0.01$ ) (the cutoff value is the median). All the above results were verified by PCR of clinical glioma samples and the follow-up results of patients in our hospital (Figures 1B,E,H), $p<0.05$ ).

\section{TXNDC12 is an Independent Prognostic Factor for Patients With Glioma}

We analyzed the relationship between TXNDC12 and the prognosis of patients with glioma by establishing a Cox regression model. Univariate analysis revealed that TXNDC12 is a significant risk factor for glioma in TCGA-RNA-seq (HR = $4.182,95 \% \mathrm{CI}=3.185-5.490, p<0.001$ ) (Figure 2A) and CGGARNA-seq $(\mathrm{HR}=2.186,95 \% \mathrm{CI}=1.943-2.460, p<0.001)$ (Figure 2D). We found that TXNDC12 is an independent risk factor for glioma in CGGA-RNA-seq by multivariate analysis $(\mathrm{HR}=1.371,95 \% \mathrm{CI}=1.188-1.584, p<0.001)$ (Figure 2E). However, the result was not statistically significant in TCGARNA-seq $(\mathrm{HR}=1.359,95 \% \mathrm{CI}=0.915-2.018, p=0.128)$ (Figure 2B). Furthermore, we found that TXNDC12 was an prognostic factor for 3-year and 5-year survival in glioma patients both in TCGA-RNA-seq $\left(\mathrm{AUC}_{3 \text { year }}=0.787, \mathrm{AUC}_{5 \text { year }}=0.755\right)$ (Figure 2C) and CGGA-RNA-seq $\left(\mathrm{AUC}_{3 \text { year }}=0.748, \mathrm{AUC}_{5 \text { year }}\right.$ $=0.774)$ (Figure $2 \mathrm{~F}$ ) by ROC curves.

To test the validity and authenticity of the analysis results from TCGA and CGGA databases applicable to actual clinical patients, we collected follow-up data of glioma patients in our hospital 
TABLE 1 | Association of TXNDC12 expression is with the clinicopathological characteristics of patients with glioma.

\begin{tabular}{|c|c|c|c|c|c|c|}
\hline \multirow[t]{2}{*}{ Clinical features } & \multirow[t]{2}{*}{ Patient number } & \multicolumn{3}{|c|}{ TXNDC12 expression } & \multicolumn{2}{|c|}{ Univariate analysis } \\
\hline & & High & Low & HR & $95 \% \mathrm{Cl}$ & $p$-value \\
\hline TXNDC12 & 25 & 13 & 12 & 1.604 & (1.178-2.182) & 0.003 \\
\hline Age & & & & & $>=48$ vs $<48$ & \\
\hline$<48$ & 12 & 6 & 6 & & reference group & \\
\hline$>=48$ & 13 & 7 & 6 & 0.82 & $(0.36-1.86)$ & 0.63 \\
\hline Gender & & & & & Male vs Female & \\
\hline Female & 11 & 5 & 6 & & reference group & \\
\hline Male & 14 & 8 & 6 & 2.15 & $(0.92-5.05)$ & 0.08 \\
\hline WHO grade & & & & & (IV or III) vs II & \\
\hline II & 8 & 3 & 5 & & reference group & \\
\hline III & 7 & 3 & 4 & 1.146 & (0.379-3.465) & 0.809 \\
\hline IV & 10 & 7 & 3 & 2.059 & (1.158-3.662) & 0.014 \\
\hline KPS score & & & & & $\geq 80$ vs $<80$ & \\
\hline$<80$ & 9 & 4 & 5 & & reference group & \\
\hline$\geq 80$ & 16 & 9 & 7 & 1.49 & $(0.62-3.55)$ & 0.37 \\
\hline Tumor location & & & & (Fronta & or Temporal lobe) vs & lobe) \\
\hline Occipital lobe & 3 & 2 & 1 & & reference group & \\
\hline Parietal lobe & 6 & 2 & 4 & 1.281 & $(0.571-2.874)$ & 0.548 \\
\hline Temporal lobe & 5 & 3 & 2 & 1.614 & (0.307-8.473) & 0.571 \\
\hline Frontal lobe & 11 & 6 & 5 & 1.582 & (0.345-7.264) & 0.555 \\
\hline Tumor volume(cm3) & & & & & $\geq 37$ vs $<37$ & \\
\hline$<37$ & 11 & 5 & 6 & & reference group & \\
\hline$\geq 37$ & 14 & 8 & 6 & 0.937 & $(0.409-2.147)$ & 0.878 \\
\hline Postsurgical radiotherapy & & & & & No vs Yes & \\
\hline Yes & 18 & 11 & 7 & & reference group & \\
\hline No & 7 & 2 & 5 & 0.502 & $(0.198-1.272)$ & 0.146 \\
\hline Postsurgical TMZ therapy & & & & & No vs Yes & \\
\hline Yes & 16 & 8 & 8 & & reference group & \\
\hline No & 9 & 5 & 4 & 0.672 & $(0.279-1.620)$ & 0.376 \\
\hline
\end{tabular}

KPS score, Karnofsky performance score; HR, Hazard Ratio; Cl, confidence interval; bold values represent statistical significance; $\mathrm{p}<0.05$ considered statistically significant.

(Table 1) for validation. The results showed that expression of TXNDC12 $(\mathrm{HR}=1.604,95 \% \mathrm{CI}=1.178-2.182, p<0.005)$ and WHO grade of glioma $(\mathrm{HR}=2.059,95 \% \mathrm{CI}=1.158-3.662, p<$ $0.05)$ were risk factors for glioma patients. However, patient age, gender, KPS (Karnofsky Performance Scale) score, and tumor location were not statistically significant.

\section{The Relationship Between TXNDC12 and 1p19q Status}

$1 \mathrm{p} 19 \mathrm{q}$ has important prognostic implications for glioma patients as a molecular marker for pathological classification. We have demonstrated that high expression of TXNDC12 in glioma predicts adverse outcomes in patients. To explore whether there is any relationship between TXNDC12 and 1p19q status, we found that the expression of TXNDC12 was significantly lower in the $1 \mathrm{p} 19 \mathrm{q}$ Codel (1p19q co-deletion) group compared to the $1 \mathrm{p} 19 \mathrm{q}$ Non-codel $(1 \mathrm{p} 19 \mathrm{q}$ non-codeletion) group, both in TCGA (Figure 3A, $p<0.005$ ) and CGGA (Figure 3E, $p<0.005$ ). The expression of TXNDC12 in the $1 \mathrm{p} 19 \mathrm{q}$ codel subgroup increased with glioma WHO grade (Figure 3F, $p<0.005$ ) in CGGA. However, this difference was not statistically significant in TCGA (Figure 3B, $p=0.073$ ). In the $1 \mathrm{p} 19 \mathrm{q}$ Non-codel subgroup, TXNDC12 expression significantly increased with the rise of the grade of gliomas in both TCGA (Figure 3C, $p<0.005$ ) and CGGA (Figure 3G, $p<0.005$ ). In the 1p19q Noncodel subtype, TCGA (Figure 3D, $p<0.005$ ) and CGGA
(Figure 3H, $p<0.005$ ), patients in the TXNDC12 high expression group had a worse prognosis than those in the TXNDC12 low expression group. In the $1 \mathrm{p} 19 \mathrm{q}$ Codeletion subtype, CGGA (Figure 3H, $p<0.005$ ), patients in the TXNDC12 high expression group had a worse prognosis than those in the TXNDC12 low expression group, while this change was not statistically significant in the TCGA database.

\section{The Relationship Between TXNDC12 and Isocitrate Dehydrogenase Status}

Similar to $1 \mathrm{p} 19 \mathrm{q}, \mathrm{IDH}$ is another glioma typing biomarker widely used clinically. We found that the expression of TXNDC12 in the IDH Wildtype group was significantly higher than that in the IDH Mutant group in TCGA (Figure 4A, $p<0.005$ ) and CGGA (Figure 4E, $p<0.005)$. In the IDH Mutant subgroups, TCGA (Figure 4B, $p<0.005$ ) and CGGA (Figure 4F, $p<0.005$ ), the expression of TXNDC12 increased significantly with the rise of glioma grade, and the same results were observed in the IDH Wildtype subgroup (Figure 4C, $p<0.005$ and Figure 4G, $p<$ 0.005 ). In the IDH Wildtype subtype, TCGA (Figure 4D, $p<$ 0.005 ) and CGGA (Figure 4H, $p<0.005$ ), patients in the TXNDC12 high expression group had a worse prognosis than those in the TXNDC12 low expression group. In the IDH Mutant subtype, CGGA (Figure $4 \mathbf{H}, p<0.005$ ), patients in the TXNDC12 high expression group had a worse prognosis than those in the TXNDC12 low expression group. However, in the TCGA, the 

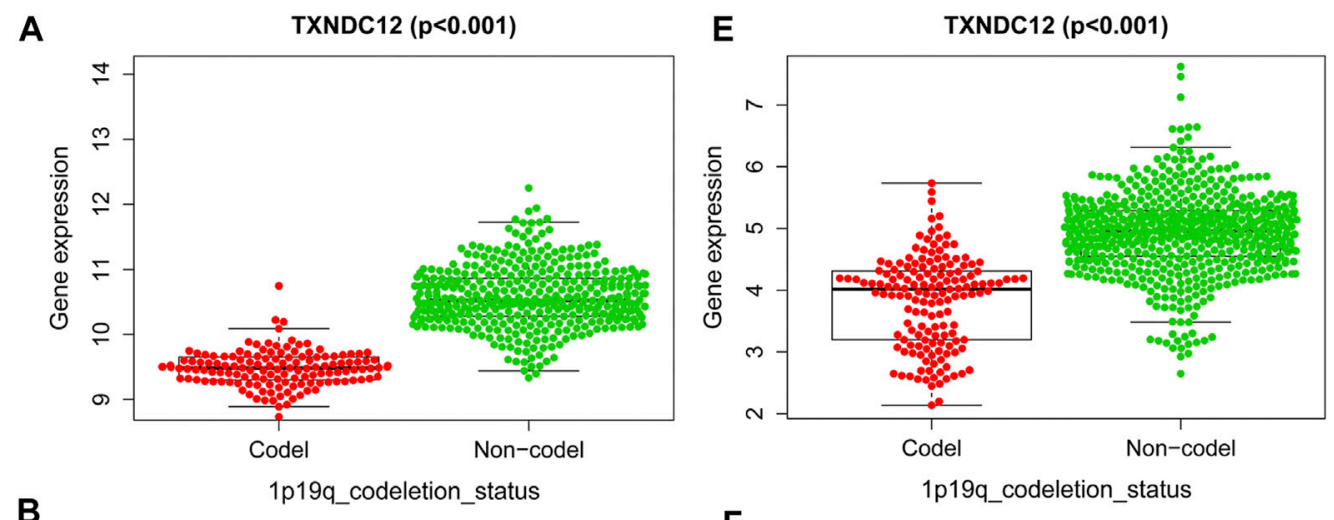

B

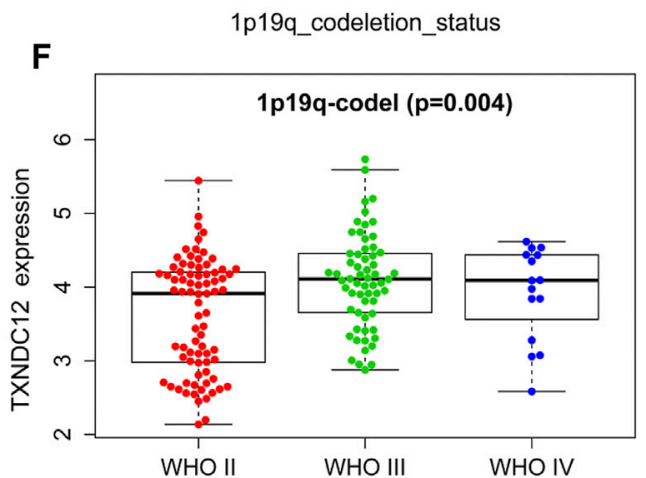

C
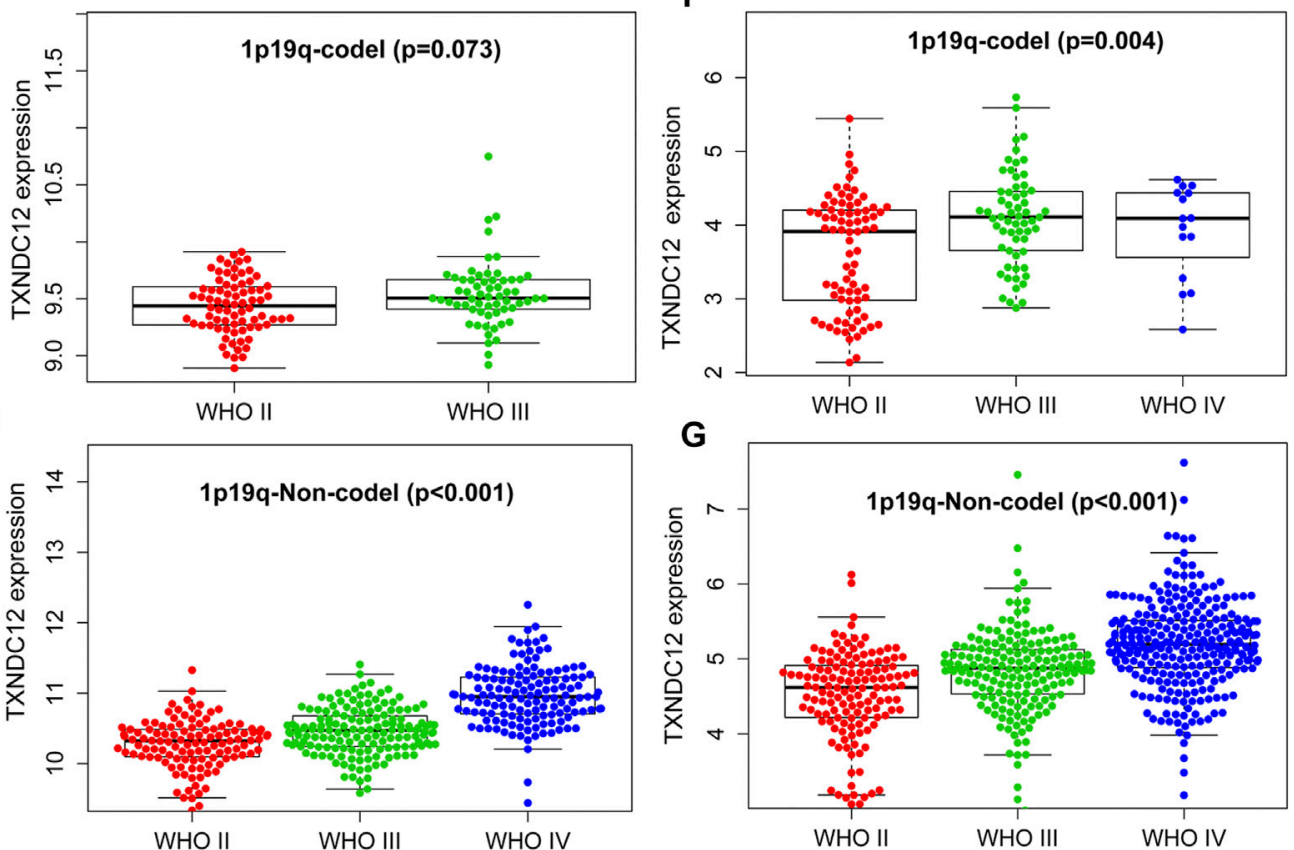

D

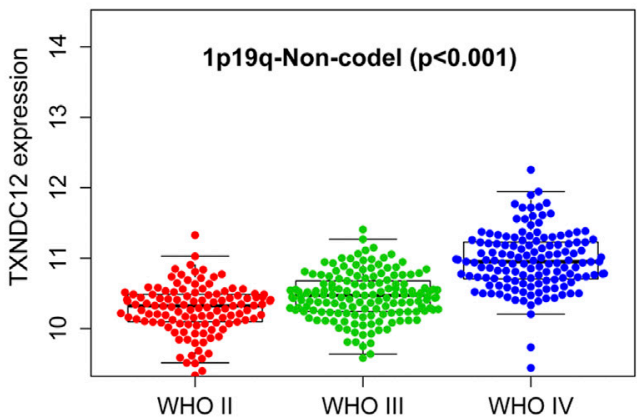

Survival curve $(\mathrm{p}=0 \mathrm{e}+00)$
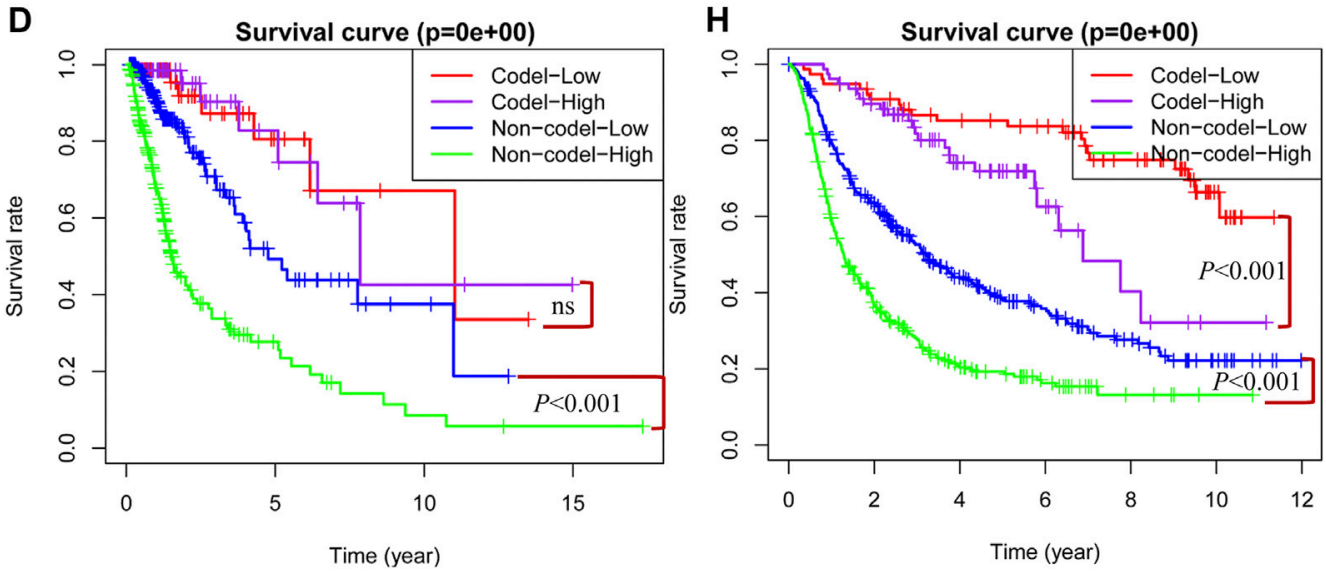

FIGURE 3 | Combined detection of 1p19q and TXNDC12 is meaningful for prognostic judgment of glioma. The difference of TXNDC12 expression in different 1p19q status gliomas from TCGA (A) and CGGA (E). The relationship between TXNDC12 expression and different glioma grade in 1p19q Codel glioma from TCGA (B) and CGGA (F). The relationship between TXNDC12 expression in 1p19q Non-codel glioma and glioma grade from TCGA (C) and CGGA (G); Survival curve for combined test of TXNDC12 and 1p19q from TCGA (D) and CGGA (H). 

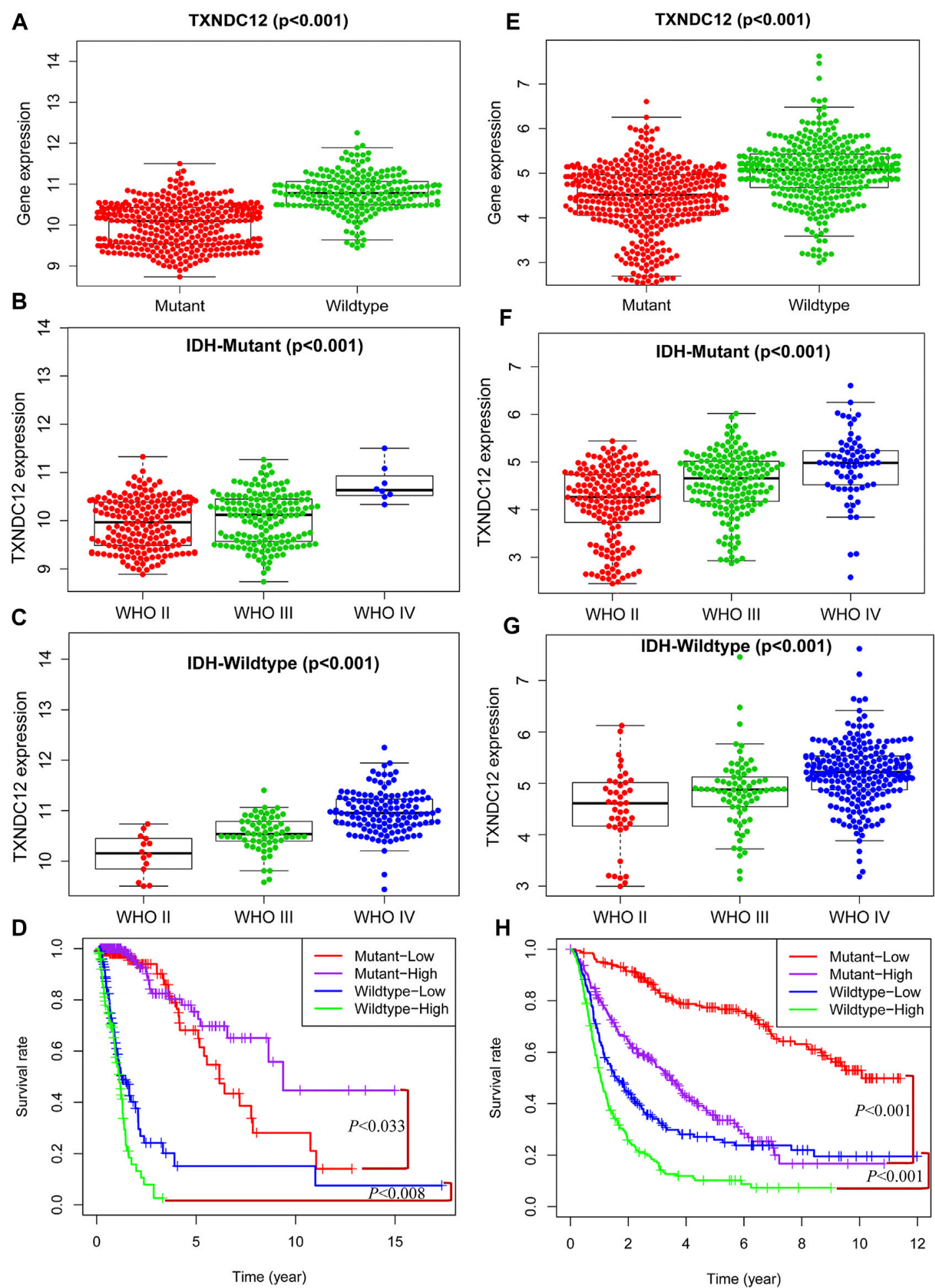

FIGURE 4 | Combined detection of IDH and TXNDC12 is meaningful for prognostic judgment of glioma. The difference of TXNDC12 expression in different IDH status gliomas from TCGA (A) and CGGA (E). The relationship between TXNDC12 expression and different glioma grade in IDH Mutant glioma from TCGA (B) and CGGA (F). The relationship between TXNDC12 expression in IDH Wildtype glioma and glioma grade from TCGA (C) and CGGA (G); Survival curve for combined test of TXNDC12 and IDH status from TCGA (D) and CGGA (H). 

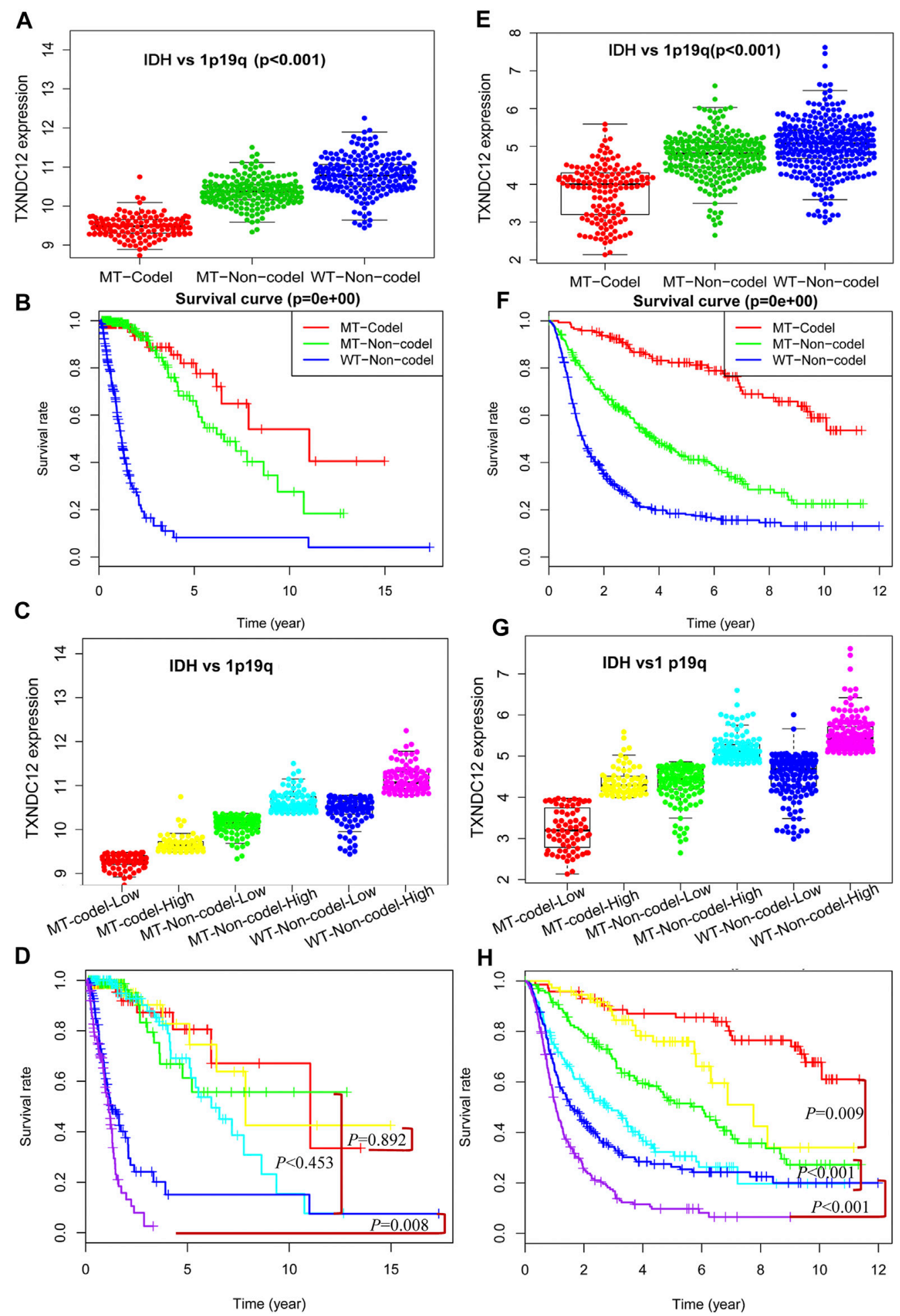

FIGURE 5 | Comprehensive analysis of TXNDC12 combined with 1p19q and IDH status on the prognosis of glioma. Detection of TXNDC12 expression, 1p19q status and IDH status in samples from TCGA (A) and CGGA (E). Survival curve of patients with three subtypes of glioma: TCGA (B) and CGGA (F); Each type of part (A) and $\mathbf{( E )}$ is divided into two subgroups in this figure (division cut point value is the median of TXNDC12 expression level). Part (C) corresponds to part (A), part (G) corresponds to part (E). Survival curves of patients with six subtypes of glioma samples from TCGA (D) and CGGA (H): The subtypes represented by different colors have the same meaning as those represented in parts (C) and (E). 

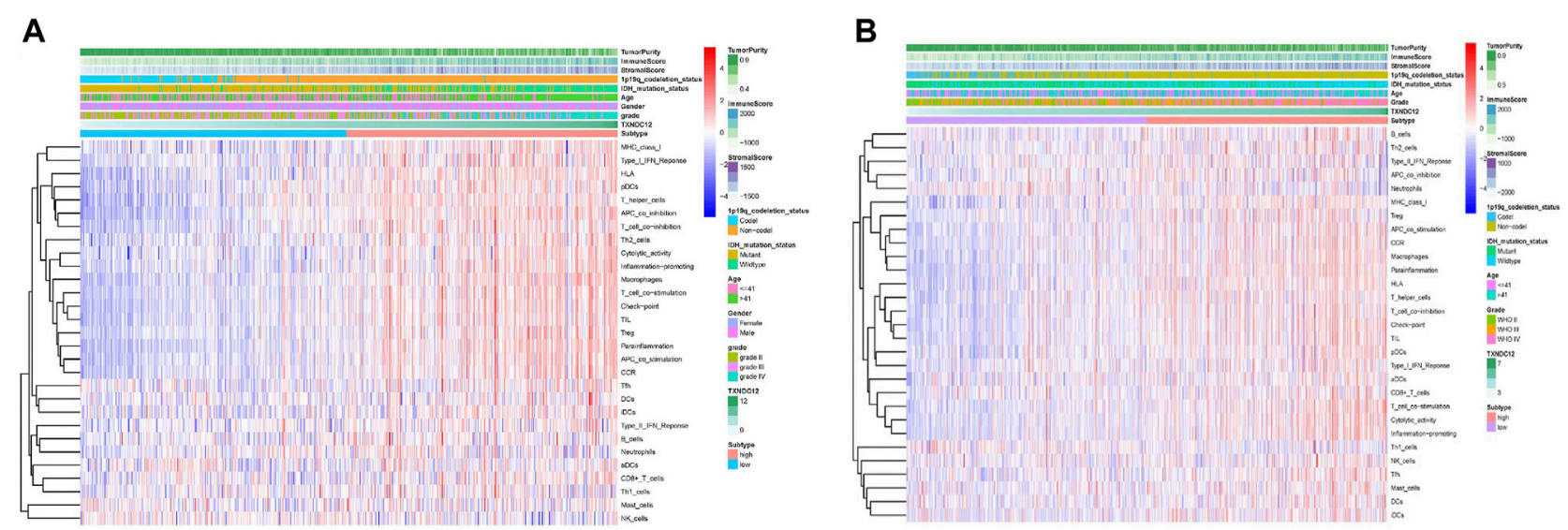

C

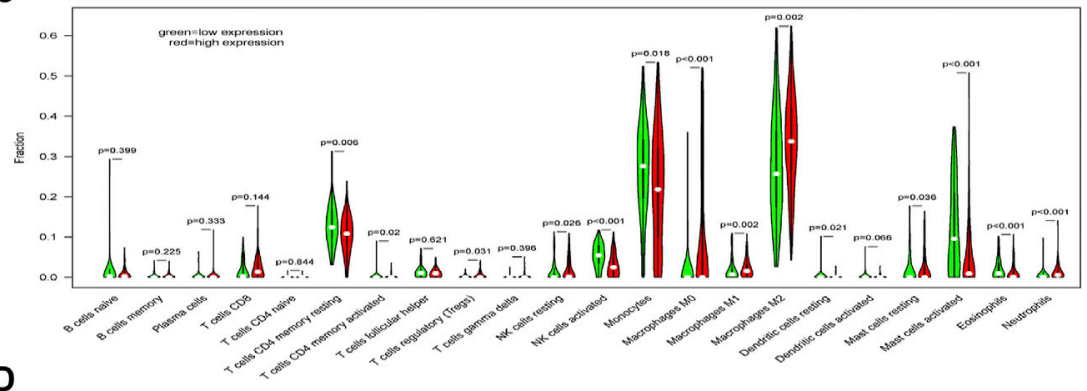

D

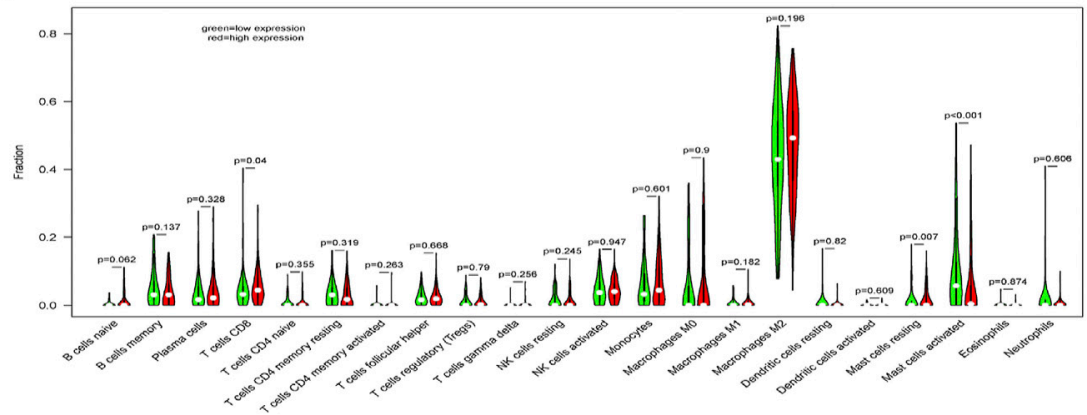

FIGURE 6 | The effect of TXNDC12 on the immune microenvironment of glioma. In TCGA (A) CGGA (B), heatmap of the patient's clinical characteristics and tumor local immune status. The infiltration of immune cells in the TXNDC12 high and low expression subgroups of glioma samples from TCGA (C) and CGGA (D).

prognosis of MT-high (IDH Mutant combined with TXNDC12 high expression) is better than that of MT-low (IDH Mutant combined with TXNDC12 low expression).

\section{The Effect of TXNDC12 Combined With IDH and 1p19q on Glioma}

In previous studies, we have proved the significance of TXNDC12 alone, TXNDC12 combined with IDH, and TXNDC12 combined with $1 \mathrm{p} 19 \mathrm{q}$ on glioma patient prognosis. However, we know that these three molecular changes may occur in the same patient in actual clinical cases. Reliant on any single molecule to analyze glioma patients' pathological condition and prognosis may be relatively weak and confusing. Therefore, we combined IDH, $1 \mathrm{p} 19 \mathrm{q}$, and TXNDC12 to explore their combined effects on patient prognosis with glioma. In Figure 5A (TCGA, $p<0.005$ ) and Figure 5E (CGGA, $p<0.005)$, we found that TXNDC12 had the lowest expression in the MT-Codel (IDH Mutation combining $1 \mathrm{p} 19 \mathrm{q}$ Codeletion) group, while the MT-Noncodel (IDH Mutant combining 1p19q Non-codeletion) was moderate, and the WT-Non-codel (IDH Wildtype combining $1 \mathrm{p} 19 \mathrm{q}$ Non-codeletion) had the highest expression. Survival analysis showed that the MT-Codel subgroup had the longest survival time, followed by the MT-Non-codel group. The WT-Non-codel group had the shortest survival time (Figure 5B, $p<0.005$; and Figure 5F, CGGA, $p<0.005$ ). To further investigate the effect of the difference in TXNDC12 expression among the three subtypes on glioma prognosis, we divided the samples of each subtype into TXNDC12 high-expression and low-expression groups according to the median TXNDC12 expression in the samples. That is, the above three groups were divided into six subgroups (Figure 5C, TCGA; and Figure 5G, CGGA), and survival curves were drawn for Figure 5D (TCGA) and 

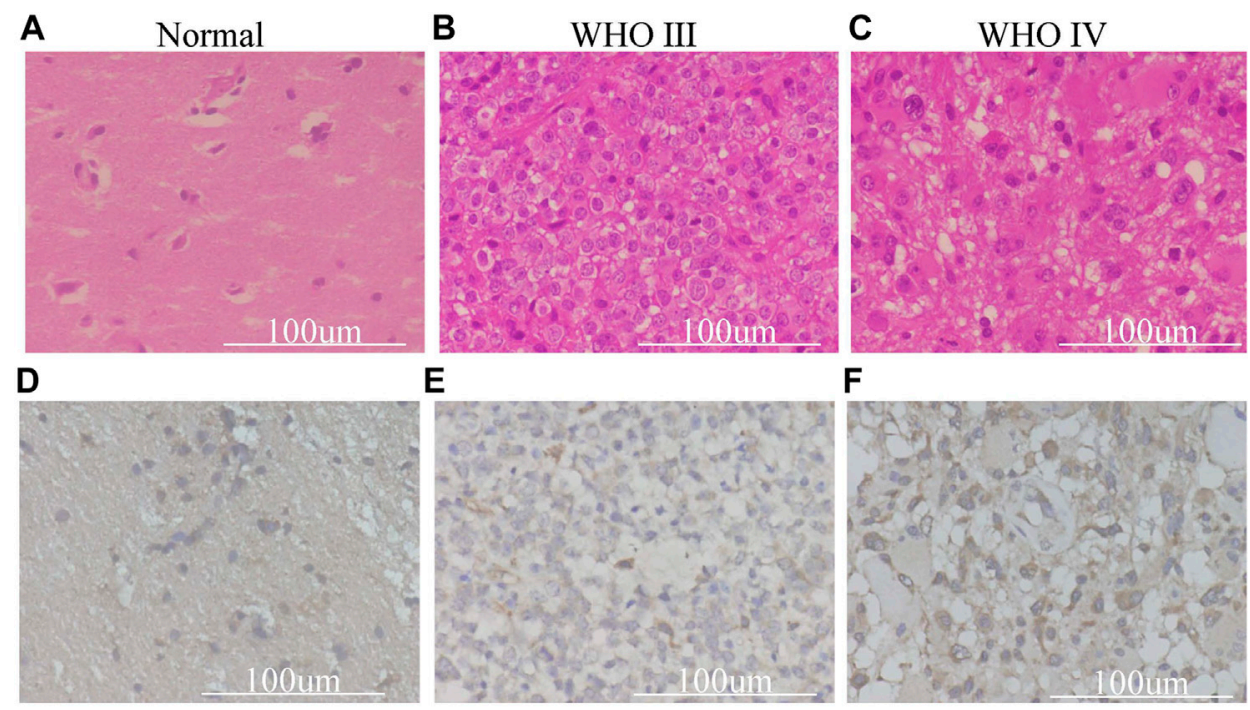

$\mathbf{F}$

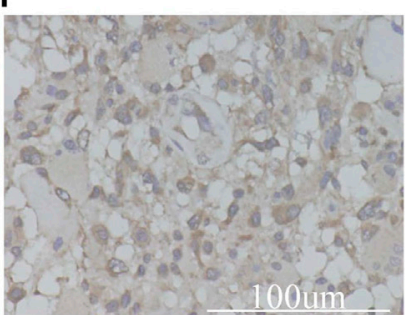

FIGURE 7 | HE staining (A-C) and Immunohistochemical (D-F) section of surgical samples in our hospital.

Figure 5H (CGGA). The results showed that TXNDC12 high expression group had shorter survival within the same subtype compared to the low expression group in CGGA (Figure 5D). The WT-Non-codel subtype also showed the same results in TCGA, but the difference in survival curves was not statistically significant between MT-codel and MTNon-codel subtypes (Figure 5H).

\section{The Relationship Between TXNDC12 and Immune Infiltration in Glioma}

We know that there is an immunosuppressive phenomenon in glioma lesions, which affects the efficacy of many therapeutic measures against glioma. To investigate the relationship between TXNDC12 and the local immune microenvironment glioma, we analyzed data from TCGA (Figure 6A) and CGGA (Figure 6B) databases. The results showed that the clinical features of gliomas in the TXNDC12 high expression group were mainly associated with IDH wild-type, 1p19q non-codeletion, age $>41$ years, higher WHO grade, reduced tumor cell purity, increased immune cells, and extracellular matrix in the tumor tissue. Further analysis of the infiltrating immune cell components revealed that infiltration of immune cells in glioma tissue is inconsistent across populations and mast cells decreased in the TXNDC12 high expression group (Figures 6C,D).

\section{The Protein Expression Level of TXNDC12 in Glioma}

We found that the combination of IDH and $1 \mathrm{p} 19 \mathrm{q}$ status has an absolute predictive value for the prognosis of glioma patients. The expression of TXNDC12 is significantly increased at the mRNA level in gliomas; however, it is unclear whether the expression of the protein encoded by the final target TXNDC12 gene also has corresponding changes. We took normal brain tissue, WHO III, and WHO IV glioma tissues for HE staining (Figures 7A-C) and performed TXNDC12 immunohistochemical analysis (Figures 7D-F,). HE staining showed that WHO III and WHO IV gliomas were dense, with large, and dark stained nuclei and irregular shape (Figures 7B,C). Immunohistochemistry showed an increase in TXNDC12-positive cells in WHO III and WHO IV glioma tissues compared to normal brain tissues (Figure 7E,F).

\section{Functional Enrichment Analysis of Genes Related to TXNDC12 and Glioma Staging}

Co-expression analysis yielded 1301 TXNDC12-related genes in TCGA and 4,374 in CGGA and intersected these genes to obtain 398 genes, as shown in Figure 8A. The GO enrichment analysis of the biological processes (Figure 8B), cellular components (Figure 8C), and molecular functions (Figure 8D) involved in these genes revealed that the functions of these genes were mainly focused on RNA synthesis, antigen presentation and processing, neutrophil-mediated immune response, and glycosylation. The KEGG analysis (Figures 8E,F) shows that major pathways are enriched in glycosyl transfer reaction and DNA transcription.

\section{DISCUSSION}

Glioma is the most common primary intracranial tumor, accounting for $75 \%$ of malignant brain tumors[9]. Although it has lower morbidity compared to tumors in other systems throughout the body, it causes a high mortality rate[22]. Among them, glioblastoma has the lowest OS rate, with only $4.7 \%$ of patients surviving within 5 years after diagnosis[23]. In recent years, emerging therapeutic modalities such as immunotherapy and Tumor Treating Fields have been 
A

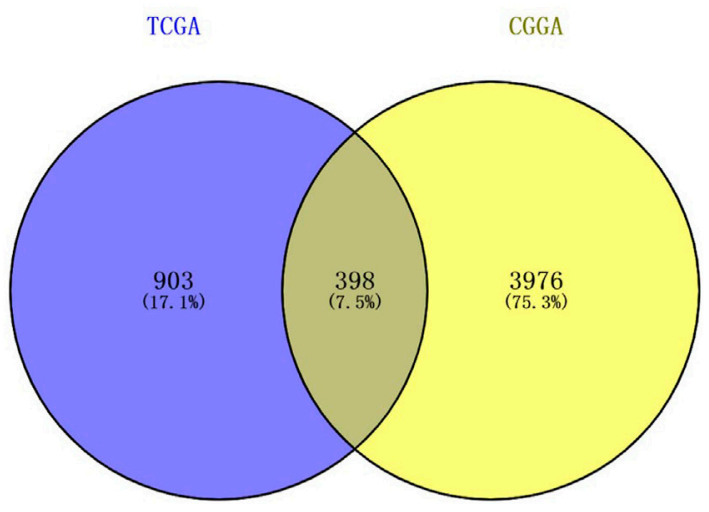

C

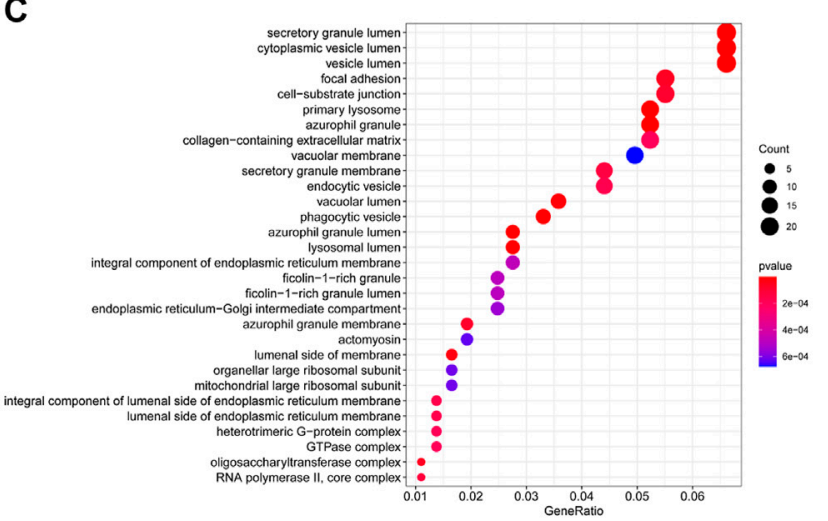

E

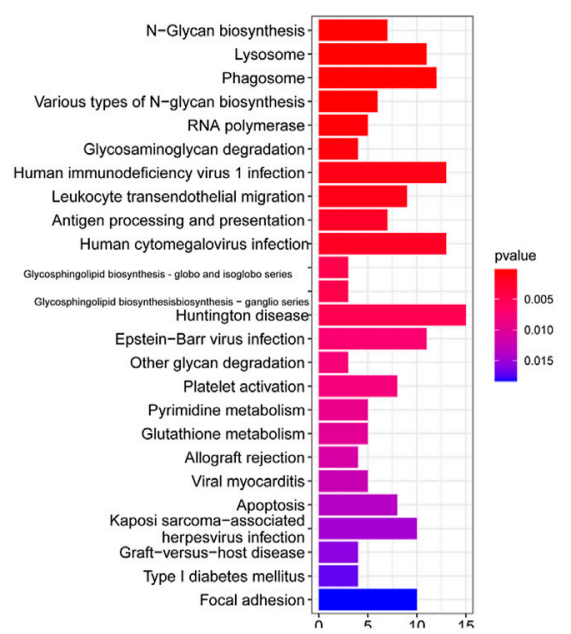

B

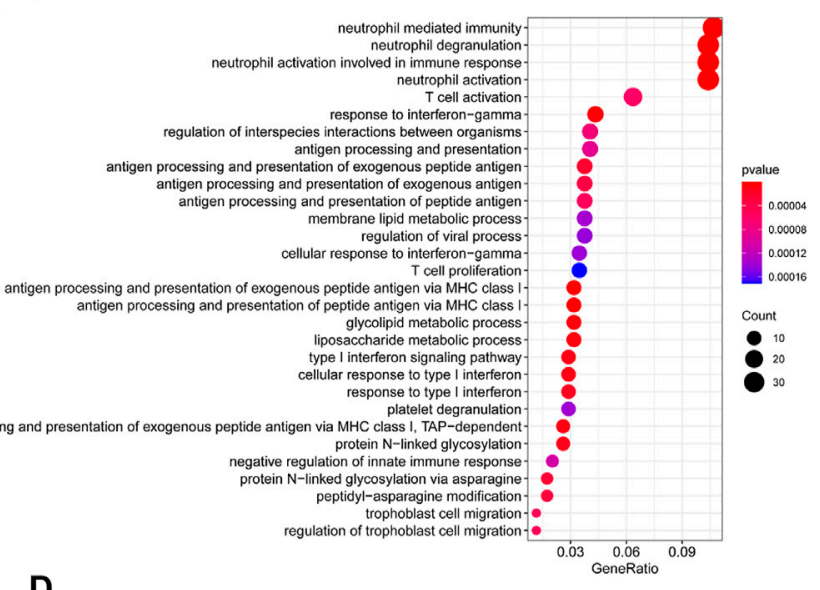

D

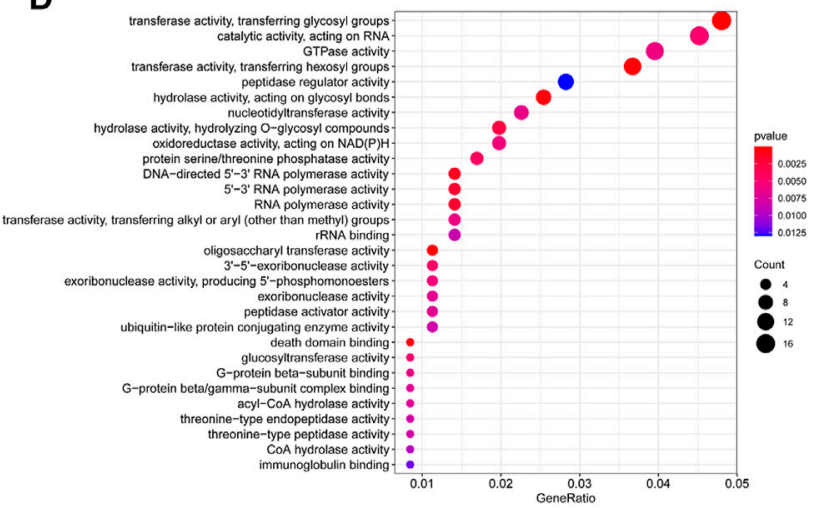

$\mathbf{F}$

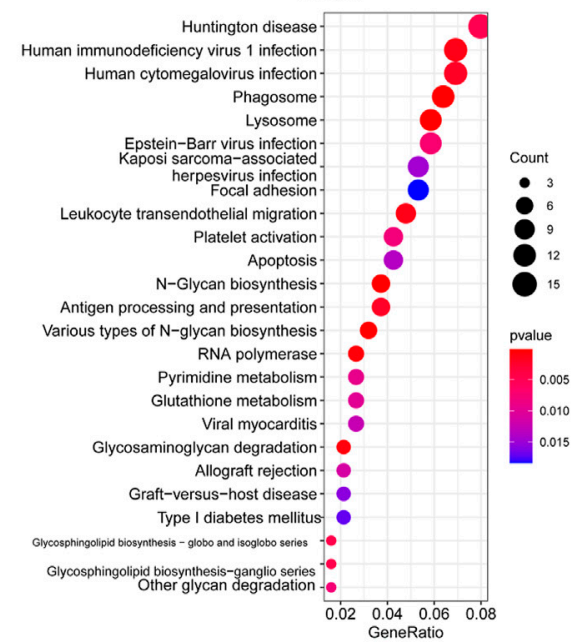

FIGURE 8 | Functional enrichment analysis of genes related to TXNDC12 and glioma staging. Intersection of TXNDC12 Co-Expressed Genes in TCGA and CGGA (A). Biological processes associated with the co-expressed gene of TXNDC12 (B). Cellular components associated with the co-expressed gene of TXNDC12 (C). Molecular functions associated with the co-expressed gene of TXNDC12 (D). KEGG analysis for genes co-expressed with TXNDC12 (E, F).

attempted to be used as treatments for gliomas, however, no breakthrough progress has been shown[24-26]. Tumors are caused by abnormal tissue cell changes at the genetic level, and gliomas are no exception[27]. Many complex pathways are involved, and the level of awareness of glioma limits the ability to diagnose and treat tumors[28,29]. Genetic therapy is a significant breakthrough in solving the problem of tumor treatment. The development of sequencing technology and bioinformatics has led to the discovery of many specific biomarkers, which are of great therapeutic value $[8,30]$.

We screened the TXNDC12 gene using public databases and found that it is highly expressed in gliomas, and the expression 
level increased significantly with the level of gliomas. The PCR results of glioma samples from our hospital verified this conclusion. Further regression analysis found that TXNDC12 is an independent risk factor for the prognosis of glioma, which has a certain clinical application value. However, in the multivariate risk analysis for TXNDC12, it was found that the analysis results for the TCGA database $(\mathrm{HR}=1.359,95 \% \mathrm{CI}=$ $0.915-2.018, p=0.128$ ) were not statistically significant. We found that this may be related to different races and sample sizes.

For many years, the pathological classification and grading of gliomas have been based on the morphological criteria of microscopic tumor tissues[30]. However, in clinical practice, it has been found that this standard does not accurately reflect the actual course of glioma and has a limited guiding value for the treatment and prognosis of patients[31]. With the discovery of the significance of molecular changes such as IDH and 1p19q in gliomas, the WHO 2016 version of the pathological classification of gliomas introduced these standards[32,33]. We combined IDH and $1 \mathrm{p} 19 \mathrm{q}$ for analysis and found that the expression level of TXNDC12 in IDH mutant type was significantly lower than that in IDH wild-type, and the expression level of the $1 \mathrm{p} 19 \mathrm{q}$ deletion type was significantly lower than that of the $1 \mathrm{p} 19 \mathrm{q}$ non-deletion type. The TXNDC12 low expression group of different subtypes had better prognosis, and the combination of these three subtypes can have a better guiding significance for the pathological classification and prognosis judgment of glioma.

In addition to tumor cells, various extracellular matrices, cytokines, and infiltrating immune cells of various types are components that together make up the glioma tumor microenvironment[34]. There is still much work to investigate the role of these immune cells in the microenvironment of glioma. However, an increasing number of researchers are discovering that the non-tumor cell components in glioma also play an important role in the occurrence and progression of glioma[35]. Many relevant research results are currently being reported. For example, tumor-associated macrophages in the tumor microenvironment are involved in forming the local immunosuppressive state in glioma[36], which explains the unsatisfactory efficacy of various current immunotherapeutic measures against glioma[37,38]. These results indicate that glioma's local immune microenvironment plays a vital role in treating glioma [39]. Therefore, we also analyzed the infiltration status of glioma immune cells in the samples. The results showed that in the group with high TXNDC12 expression, tumor cell purity in tumor tissues decreased, immune cells, and extracellular matrix increased, and mast cell activity decreased. Some researchers found that glioma tissues induce migration of mast cells to glioma tissues by producing cytokines such as glioma-derived macrophage mobility inhibitory factor[40]. These mast cells reduce the proliferative and invasive capacity of glioma cells and induce their differentiation by depleting the potency of glioma stem cells, possibly by inactivating the STAT3-mediated pathway through GSK3 $\beta$ downregulation[41]. Based on the above results, we infer that mast cells may be involved in the generation of poor prognosis in patients with TXNDC12 highexpressing glioma.

We found that TXNDC12 is involved in the poor prognosis of glioma. To further investigate the mechanism by which TXNDC12 is involved in the malignant prognosis of glioma, we analyzed the expression of TXNDC12 protein in glioma and normal brain tissue and found that TXNDC12 protein expression was significantly increased in gliomas and appeared to be positively correlated with glioma grade. Unfortunately, due to the small number of clinical glioma samples we collected for immunohistochemical analysis (especially for grade II gliomas), we were unable to perform a valid statistical analysis of the differences in TXNDC12 expression in different gliomas as well as in normal brain tissue, which is a drawback of this study. Therefore, we hypothesized that TXNDC12 is involved in the malignant progression of gliomas through the expression of corresponding functional proteins. In addition, functional enrichment analysis of TXNDC12 functionally related genes revealed that the functions of these genes are mainly focused on the regulation of the activities of various glycosyltransferases such as glucosyltransferase and oligosaccharyltransferase, as well as in the process of RNA synthesis. The role of glycosyltransferases in glioma has also been extensively studied in several studies that have found glycosyltransferases to be associated with the development and aggressive growth of various malignant tumors such as breast cancer, glioblastomas, and glioma[42-44]. A small molecule inhibitor of N-acetylglucosaminyltransferase (MGAT5)[45], PST3.1a, can significantly inhibit the invasive and proliferative capacity of glioma by inhibiting the signaling of $\beta \mathrm{R}$ and FAKrelated pathways, and in combination with TMZ can significantly prolong the survival of glioma patients[46]. Therefore, we speculate that TXNDC12 is likely to confer enhanced proliferation and invasiveness to this type of glioma by increasing the activity of related glycosyltransferases. If our hypothesis is confirmed, the development of new drugs targeting this glycosyltransferase enzyme is expected to bring new solutions to glioma drug therapy and provide an effective complement to current temozolomide therapy, which is plagued by widespread drug resistance problems. However, as one of the shortcomings of this experiment, we did not design a validation experiment to confirm our conjecture.

\section{CONCLUSION}

We analyzed the vital role of the TXNDC12 gene in glioma and showed that TXNDC12 is highly expressed in glioma tissue and that it is significantly related to pathological grade and poor prognosis. So, TXNDC12 may serve as a potential molecular marker for glioma pathological grade and prognosis.

\section{DATA AVAILABILITY STATEMENT}

The raw data supporting the conclusions of this article will be made available by the authors, without undue reservation.

\section{ETHICS STATEMENT}

The studies involving human participants were reviewed and approved by First Affiliated Hospital of Harbin Medical 
University. The patients/participants provided their written informed consent to participate in this study.

\section{AUTHOR CONTRIBUTIONS}

QY generated the hypothesis, collected, and analyzed the data; XW analyzed the data; NL wrote and edited the manuscript; QB reviewed the manuscript; MG provided software technical support and verified the analysis results; $\mathrm{XH}$ provided financial support and reviewed the manuscript.

\section{FUNDING}

National Natural Science Foundation of China (No. 81402062) National Natural Science Foundation of China (No. 81371301).

\section{REFERENCES}

1. Ostrom QT, Patil N, Cioffi G, Waite K, Kruchko C, and Barnholtz-Sloan JS. CBTRUS Statistical Report: Primary Brain and Other Central Nervous System Tumors Diagnosed in the United States in 2013-2017. Neuro Oncol (2020) 22(12 Suppl. 2):iv1-iv96. doi:10.1093/neuonc/noaa200

2. Wen PY, and Kesari S. Malignant Gliomas in Adults. N Engl J Med (2008) 359(5):492-507. doi:10.1056/NEJMra0708126

3. Brat DJ, Verhaak RG, Aldape KD, Yung WKA, Salama SR, Cooper LAD, et al. Comprehensive, Integrative Genomic Analysis of Diffuse Lower-Grade Gliomas. N Engl J Med (2015) 372(26):2481-98. doi:10.1056/NEJMoa1402121

4. Youssef G, and Miller JJ. Lower Grade Gliomas. Curr Neurol Neurosci Rep (2020) 20(7):21. doi:10.1007/s11910-020-01040-8

5. Delgado-Martín B, and Medina MÁ. Advances in the Knowledge of the Molecular Biology of Glioblastoma and its Impact in Patient Diagnosis, Stratification, and Treatment. Adv Sci (2020) 7(9):1902971. doi:10.1002/ advs.201902971

6. Mair MJ, Geurts M, van den Bent MJ, and Berghoff AS. A Basic Review on Systemic Treatment Options in WHO Grade II-III Gliomas. Cancer Treat Rev (2020) 92:102124. doi:10.1016/j.ctrv.2020.102124

7. Louis DN, Perry A, Reifenberger G, von Deimling A, Figarella-Branger D, Cavenee WK, et al. The 2016 World Health Organization Classification of Tumors of the Central Nervous System: A Summary. Acta Neuropathol (2016) 131(6):803-20. doi:10.1007/s00401-016-1545-1

8. Molinaro AM, Taylor JW, Wiencke JK, and Wrensch MR. Genetic and Molecular Epidemiology of Adult Diffuse Glioma. Nat Rev Neurol (2019) 15(7):405-17. doi:10.1038/s41582-019-0220-2

9. Lapointe S, Perry A, and Butowski NA. Primary Brain Tumours in Adults. The Lancet (2018) 392(10145):432-46. doi:10.1016/S0140-6736(18)30990-5

10. Eckel-Passow JE, Lachance DH, Molinaro AM, Walsh KM, Decker PA, Sicotte $\mathrm{H}$, et al. Glioma Groups Based on 1p/19q,IDH, andTERTPromoter Mutations in Tumors. N Engl J Med (2015) 372(26):2499-508. doi:10.1056/NEJMoa1407279

11. Chen R, Smith-Cohn M, Cohen AL, and Colman H. Glioma Subclassifications and Their Clinical Significance. Neurotherapeutics (2017) 14(2):284-97. doi:10.1007/s13311-017-0519-x

12. Jeong W, Lee D-Y, Park S, and Rhee SG. ERp16, an Endoplasmic ReticulumResident Thiol-Disulfide Oxidoreductase. J Biol Chem (2008) 283(37): 25557-66. doi:10.1074/jbc.M803804200

13. Liu F, Rong Y-P, Zeng L-C, Zhang X, and Han Z-G. Isolation and Characterization of a Novel Human Thioredoxin-Like Gene hTLP19 Encoding a Secretory Protein. Gene (2003) 315:71-8. doi:10.1016/s03781119(03)00732-7

14. Galligan JJ, and Petersen DR. The Human Protein Disulfide Isomerase Gene Family. Hum Genomics (2012) 6(1):6. doi:10.1186/1479-7364-6-6

\section{CONFLICT OF INTEREST}

The authors declare that the research was conducted in the absence of any commercial or financial relationships that could be construed as a potential conflict of interest.

\section{ACKNOWLEDGMENTS}

We thank all members of the Institute of Brain Science of Harbin Medical University for their help with this study.

\section{SUPPLEMENTARY MATERIAL}

The Supplementary Material for this article can be found online at: https://www.por-journal.com/articles/10.3389/pore.2021.1609825/ full\#supplementary-material

15. Zhang J, Li X, Han X, Liu R, and Fang J. Targeting the Thioredoxin System for Cancer Therapy. Trends Pharmacol Sci (2017) 38(9):794-808. doi:10.1016/ j.tips.2017.06.001

16. Clarke HJ, Chambers JE, Liniker E, and Marciniak SJ. Endoplasmic Reticulum Stress in Malignancy. Cancer Cell (2014) 25(5):563-73. doi:10.1016/j.ccr.2014.03.015

17. Wang M, and Kaufman RJ. The Impact of the Endoplasmic Reticulum Protein-Folding Environment on Cancer Development. Nat Rev Cancer (2014) 14(9):581-97. doi:10.1038/nrc3800

18. Wu J, Chen X-H, Wang X-Q, Yu Y, Ren J-M, Xiao Y, et al. ERp19 Contributes to Tumorigenicity in Human Gastric Cancer by Promoting Cell Growth, Migration and Invasion. Oncotarget (2015) 6(14):11794-805. doi:10.18632/ oncotarget.3649

19. Lee E-K, Cho H, and Kim C-W. Proteomic Analysis of Cancer Stem Cells in Human Prostate Cancer Cells. Biochem Biophysical Res Commun (2011) 412(2):279-85. doi:10.1016/j.bbrc.2011.07.083

20. Yuan K, Xie K, Lan T, Xu L, Chen X, Li X, et al. TXNDC12 Promotes EMT and Metastasis of Hepatocellular Carcinoma Cells via Activation of ß-catenin. Cell Death Differ (2020) 27(4):1355-68. doi:10.1038/s41418 019-0421-7

21. Zheng T, Chen K, Zhang X, Feng H, Shi Y, Liu L, et al. Knockdown of TXNDC9 Induces Apoptosis and Autophagy in Glioma and Mediates Cell Differentiation by P53 Activation. Aging (2020) 12(18):18649-59. doi:10.18632/aging.103915

22. Cutts J, Brookhouser N, and Brafman DA. Generation of Regionally Specific Neural Progenitor Cells (NPCs) and Neurons from Human Pluripotent Stem Cells (hPSCs). Methods Mol Biol (2016) 1516:121-44. doi:10.1007/ 7651_2016_357

23. Omuro A, and DeAngelis LM. Glioblastoma and Other Malignant Gliomas. Jama (2013) 310(17):1842-50. doi:10.1001/jama.2013.280319

24. Lim M, Xia Y, Bettegowda C, and Weller M. Current State of Immunotherapy for Glioblastoma. Nat Rev Clin Oncol (2018) 15(7):422-42. doi:10.1038/ s41571-018-0003-5

25. Rahman M, Sawyer WG, Lindhorst S, Deleyrolle LP, Harrison JK, Karachi A et al. Adult Immuno-Oncology: Using Past Failures to Inform the Future. Neuro Oncol (2020) 22(9):1249-61. doi:10.1093/neuonc/noaa116

26. Tan AC, Ashley DM, López GY, Malinzak M, Friedman HS, and Khasraw M. Management of Glioblastoma: State of the Art and Future Directions. CA A Cancer J Clin (2020) 70(4):299-312. doi:10.3322/caac.21613

27. Gusyatiner O, and Hegi ME. Glioma Epigenetics: From Subclassification to Novel Treatment Options. Semin Cancer Biol (2018) 51:50-8. doi:10.1016/ j.semcancer.2017.11.010

28. Bi J, Chowdhry S, Wu S, Zhang W, Masui K, and Mischel PS. Altered Cellular Metabolism in Gliomas - an Emerging Landscape of Actionable Codependency Targets. Nat Rev Cancer (2020) 20(1):57-70. doi:10.1038/ s41568-019-0226-5 
29. Jonsson P, Lin AL, Young RJ, DiStefano NM, Hyman DM, Li BT, et al. Genomic Correlates of Disease Progression and Treatment Response in Prospectively Characterized Gliomas. Clin Cancer Res (2019) 25(18): 5537-47. doi:10.1158/1078-0432.Ccr-19-0032

30. Reuss DE, Mamatjan Y, Schrimpf D, Capper D, Hovestadt V, Kratz A, et al. IDH Mutant Diffuse and Anaplastic Astrocytomas Have Similar Age at Presentation and Little Difference in Survival: a Grading Problem for WHO. Acta Neuropathol (2015) 129(6):867-73. doi:10.1007/s00401-015-1438-8

31. Foote MB, Papadopoulos N, and Diaz LA, Jr. Genetic Classification of Gliomas: Refining Histopathology. Cancer Cell (2015) 28(1):9-11. doi:10.1016/j.ccell.2015.06.014

32. van den Bent MJ, Weller M, Wen PY, Kros JM, Aldape K, and Chang S. A Clinical Perspective on the 2016 WHO Brain Tumor Classification and Routine Molecular Diagnostics. Neuro Oncol (2017) 19(5):614-24. doi:10.1093/neuonc/now277

33. Reifenberger G, Wirsching H-G, Knobbe-Thomsen CB, and Weller M. Advances in the Molecular Genetics of Gliomas - Implications for Classification and Therapy. Nat Rev Clin Oncol (2017) 14(7):434-52. doi:10.1038/nrclinonc.2016.204

34. Quail DF, and Joyce JA. The Microenvironmental Landscape of Brain Tumors. Cancer Cell (2017) 31(3):326-41. doi:10.1016/j.ccell.2017.02.009

35. Klemm F, Maas RR, Bowman RL, Kornete M, Soukup K, Nassiri S, et al. Interrogation of the Microenvironmental Landscape in Brain Tumors Reveals Disease-Specific Alterations of Immune Cells. Cell (2020) 181(7):1643-60. doi:10.1016/j.cell.2020.05.007

36. Pinton L, Masetto E, Vettore M, Solito S, Magri S, D’Andolfi M, et al. The Immune Suppressive Microenvironment of Human Gliomas Depends on the Accumulation of Bone Marrow-Derived Macrophages in the center of the Lesion. J Immunotherapy Cancer (2019) 7(1):58. doi:10.1186/s40425-019-0536-x

37. Saha D, Martuza RL, and Rabkin SD. Macrophage Polarization Contributes to Glioblastoma Eradication by Combination Immunovirotherapy and Immune Checkpoint Blockade. Cancer Cell (2017) 32(2):253-67. doi:10.1016/ j.ccell.2017.07.006

38. Zhang P, Miska J, Lee-Chang C, Rashidi A, Panek WK, An S, et al. Therapeutic Targeting of Tumor-Associated Myeloid Cells Synergizes with Radiation Therapy for Glioblastoma. Proc Natl Acad Sci USA (2019) 116(47): 23714-23. doi:10.1073/pnas.1906346116
39. Sampson JH, Gunn MD, Fecci PE, and Ashley DM. Brain Immunology and Immunotherapy in Brain Tumours. Nat Rev Cancer (2020) 20(1):12-25. doi:10.1038/s41568-019-0224-7

40. Roy A, Coum A, Marinescu VD, Põlajeva J, Smits A, Nelander S, et al. GliomaDerived Plasminogen Activator Inhibitor-1 (PAI-1) Regulates the Recruitment of LRP1 Positive Mast Cells. Oncotarget (2015) 6(27):23647-61. doi:10.18632/ oncotarget. 4640

41. Attarha S, Roy A, Westermark B, and Tchougounova E. Mast Cells Modulate Proliferation, Migration and Stemness of Glioma Cells through Downregulation of GSK3 $\beta$ Expression and Inhibition of STAT3 Activation. Cell Signal (2017) 37:81-92. doi:10.1016/j.cellsig.2017.06.004

42. Veillon L, Fakih C, Abou-El-Hassan H, Kobeissy F, and Mechref Y. Glycosylation Changes in Brain Cancer. ACS Chem Neurosci (2018) 9(1): 51-72. doi:10.1021/acschemneuro.7b00271

43. Lan J, Guo P, Lin Y, Mao Q, Guo L, Ge J, et al. Role of Glycosyltransferase PomGnT1 in Glioblastoma Progression. Neuro-Oncology (2015) 17(2):211-22. doi:10.1093/neuonc/nou151

44. Dusoswa SA, Verhoeff J, Abels E, Méndez-Huergo SP, Croci DO, Kuijper LH, et al. Glioblastomas Exploit Truncated O-Linked Glycans for Local and Distant Immune Modulation via the Macrophage Galactose-type Lectin. Proc Nat Acad Sci USA (2020) 117(7):3693-703. doi:10.1073/pnas.1907921117

45. Hassani Z, Saleh A, Turpault S, Khiati S, Morelle W, Vignon J, et al. Phostine PST3.1a Targets MGAT5 and Inhibits Glioblastoma-Initiating Cell Invasiveness and Proliferation. Mol Cancer Res (2017) 15(10):1376-87. doi:10.1158/1541-7786.Mcr-17-0120

46. Li Y, Liu Y, Zhu H, Chen X, Tian $M$, Wei Y, et al. Nacetylglucosaminyltransferase I Promotes Glioma Cell Proliferation and Migration through Increasing the Stability of the Glucose Transporter GLUT1. FEBS Lett (2020) 594(2):358-66. doi:10.1002/1873-3468.13596

Copyright (c) 2021 Wang, Yang, Liu, Bian, Gao and Hou. This is an open-access article distributed under the terms of the Creative Commons Attribution License (CC $B Y)$. The use, distribution or reproduction in other forums is permitted, provided the original author(s) and the copyright owner(s) are credited and that the original publication in this journal is cited, in accordance with accepted academic practice. No use, distribution or reproduction is permitted which does not comply with these terms. 